SHORT REPORT

\title{
Reversal of glandular polarity in the lymphovascular compartment of breast cancer
}

\author{
S A Adams, M E F Smith, G P Cowley, L A Carr
}

J Clin Pathol 2004;57:1114-1117. doi: 10.1136/jicp.2004.016980

Aim: To investigate the polarity of breast invasive ductal carcinoma cells by comparing the polarity of the tumour located within lymphovascular spaces with that located in the extravascular compartment.

Methods: An immunohistochemical study identifying the apical HMFG-1, basolateral AUA-1, and basal laminin polarity markers of 11 cases of invasive ductal carcinoma (grades 1 or 2) metastatic to lymph nodes, all of which contained areas of tumour within and outside of lymphovascular spaces.

Results: Only one of 11 tumours had a focus of apparent reversed glandular polarity in the larger extravascular tumour compartment (with AUA-1 present internally and HMFG-1 expressed externally on tumour clumps), but six of the 11 tumours showed reversed glandular polarity (either with AUA-1, or HMFG-1, or both) within the very much smaller lymphovascular space tumour compartment. Laminin was not identified in association with lymphovascular tumour.

Conclusions: Reversed glandular polarity in invasive ductal breast carcinomas was identified and was significantly more frequent within vessels than outside of them. Reversal of tumour glandular polarity within lymphovascular spaces allows direct interaction between apical domain-type molecules - which are then aberrantly expressed on the external surface of tumour clumps-and lymphovascular endothelium. Such interactions may affect the establishment of metastatic disease.

A lthough the intravascular/intralymphatic (lymphovascular) tumour compartment usually constitutes only a small proportion of total tumour bulk, it represents a crucial phase in tumour spread. The redistribution of antigens to the external surfaces of tumour cell groups within lymphovascular spaces may help to determine binding between tumour cells and the vascular endothelial wall and may therefore be of importance in the establishment of metastatic disease. As part of an ongoing investigation of the properties of lymphovascular carcinoma, ${ }^{1}$ we aimed to investigate the pattern of both apical and basolateral domain markers and basal lamina in metastatic nodal breast carcinoma, comparing cancer within lymphovascular spaces with cancer outside of lymphovascular spaces.

\footnotetext{
"Redistribution of antigens to the external surfaces of tumour cell groups within lymphovascular spaces may help to determine binding between tumour cells and the vascular endothelial wall and may therefore be of importance in the establishment of metastatic disease"
}

\section{MATERIALS AND METHODS}

Eleven cases of grade I or II invasive ductal breast carcinoma arising in women were identified with metastatic tumour present in both the sinuses/vessels (lymphovascular tumour) and in the stroma of lymph nodes. Lymph node metastases were studied because of the presence of substantial lymphovascular tumour for study. Grade I/II tumours were studied because the polarity of these tumours is usually better preserved than in grade III tumours. A standard immunohistochemical avidin-biotin technique (Dako, Glostrup, Denmark) was used to immunostain paraffin wax embedded sections of these tumours using the following primary antibodies recognising glandular polarity markers and endothelium: (1) mouse monoclonal antibody AUA-1, raised against a human colonic adenocarcinoma cell line LoVo, which binds to the basolateral membrane domain of polarised epithelial glandular cells, ${ }^{2}$ purchased from Bionostics (Wyboston, Bedfordshire, UK); (2) mouse monoclonal antibody HMFG-1, raised against a component of the human milk fat globule membrane, which binds predominantly to the apical membrane domain of polarised glandular epithelial cells, ${ }^{3}$ also purchased from Bionostics; (3) rabbit antimouse laminin (polyclonal), purchased from Sigma (St Louis, Missouri, USA); and (4) mouse monoclonal antibody directed against CD31 (clone number JC/70A), purchased from Dako. It was hoped that CD31 would aid the recognition of vessels containing tumour.

\section{RESULTS}

\section{Patient group}

A histological review identified 11 cases of grade I/II invasive ductal breast carcinoma that contained appreciable amounts of extravascular and lymphovascular tumour components within the lymph node (fig 1A). None of the 11 tumours had an invasive micropapillary or mucoid morphology component. The lymphovascular tumour always constituted only a small proportion of the total nodal tumour bulk, usually much less than $10 \%$. The lymphovascular tumour included tumour within lymph node sinuses and tumour within probable angiogenetic vasculature associated with nodal metastasis.

\section{Immunohistochemistry \\ AUA- 1}

Control breast tissue showed staining of variable strength in breast lobules, some of which were negative. However, where present, the staining was always of the basolateral type, without apical staining.

Two of the 11 cases of ductal carcinoma metastatic to lymph nodes showed no AUA-1 staining and nine showed some staining in lymphovascular and/or extravascular components (table 1), although this was often weak and focal. In five of these nine cases the lymphovascular tumour component showed a focal, luminal pattern of staining, with absent peripheral staining (fig 1B), consistent with reversal 

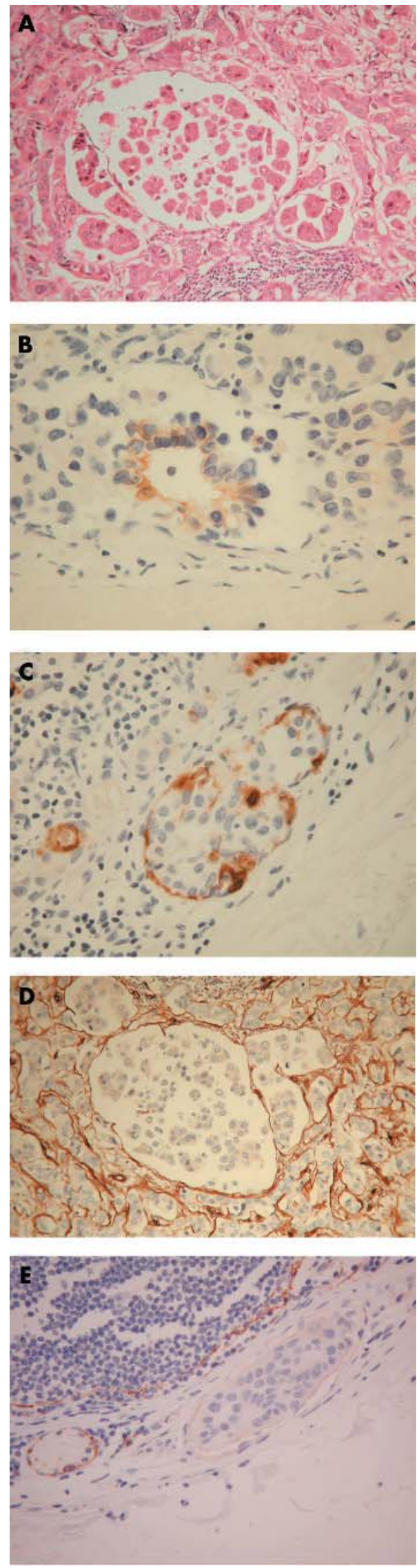

of normal glandular polarity. In the extravascular compartment such reversed glandular polarity was seen in one tumour only.

\section{HMFG- 1}

Control breast tissue showed strong staining of the luminal domain of ductal and acinar epithelial cells.

Ten of the 11 cases of metastatic ductal carcinoma showed some tumour expression of HMFG-1, albeit often weak and focal (table 1). In nine cases HMFG-1 was detected in both lymphovascular and extravascular components. Of the nine cases showing some staining of the lymphovascular tumour, four showed peripheral staining of tumour clumps in focal areas, consistent with reversed glandular polarity (fig IC). One of these cases also showed reversed polarity in the extravascular compartment (the same case that had shown reversed polarity in the extravascular compartment using AUA-1).

\section{Statistical analysis}

Combining the AUA-1 and HMFG-1 data, six of 11 cases showed reversal of apparent tumour glandular polarity in the intravascular compartment compared with one of 11 cases in the extravascular compartment. The greater frequency of tumour glandular polarity reversal within vessels compared with the outside of vessels was significant ( $p=0.027$, Fisher exact test).

\section{Laminin}

In the control breast tissue laminin identified the basal lamina of breast epithelium and endothelium.

No tumour associated laminin was detected in the lymphovascular compartment of the 11 tumour cases, despite its presence in eight cases in the extravascular compartment (fig 1D). In all 11 cases, laminin was associated with blood vessel endothelium (internal positive control).

\section{CD3 1}

In a control lymph node, CD31 delineated the subcapsular and intranodal sinusoids, in addition to blood vessels. In the tumours, although CD31 was generally useful in identifying tumour within vessels, in five of 11 cases the endothelial lining of some unequivocal lymphovascular spaces containing tumour showed a decrease in the intensity of CD31 staining compared with that seen in adjacent vessels devoid of tumour (table 1; cases $1-4$ and 11; fig $1 \mathrm{E}$ ). The remaining six cases showed no difference in endothelial staining in the presence or absence of tumour.

\section{DISCUSSION}

Our preliminary study has demonstrated immunohistochemical features consistent with a reversal of normal glandular polarity in the tumour cells of invasive ductal breast carcinoma. This reversal of tumour glandular polarity occurred significantly more frequently in lymphovascular space cancer than in extravascular space cancer, suggesting

Figure 1 (A) Haematoxylin and eosin stain showing nodal metastatic ductal carcinoma of the breast within both the extravascular and lymphovascular compartments of the lymph node. (B) Immunohistochemical staining of lymphovascular tumour with AUA-1 showing a luminal pattern - that is, reversal of polarity. (C) Immunohistochemical staining of lymphovascular tumour with HMFG-1 showing peripheral staining - that is, reversal of polarity. (D) Immunohistochemical staining of lymphovascular tumour with antilaminin showing an absence of reactivity. (E) Immunohistochemical staining with anti-CD31 showing decreased staining of the endothelium associated with the tumour compared with uniform staining of endothelial cells in an adjacent vessel with no tumour. 
Table 1 Immunostaining with antibodies AUA-1 and HMFG-1 to assess cell polarity in the extravascular (EV) and lymphovascular (LV) tumour compartments

\begin{tabular}{|c|c|c|c|c|c|c|c|c|c|c|c|c|c|c|c|c|}
\hline \multirow[b]{3}{*}{ Case } & \multicolumn{8}{|c|}{ AUA-1 } & \multicolumn{8}{|c|}{ HMFG-1 } \\
\hline & \multicolumn{2}{|c|}{ Inverse polarity } & \multicolumn{2}{|c|}{ Normal polarity } & \multicolumn{2}{|c|}{ No polarity } & \multicolumn{2}{|c|}{ No staining } & \multicolumn{2}{|c|}{ Inverse polarity } & \multicolumn{2}{|c|}{ Normal polarity } & \multicolumn{2}{|c|}{ No polarity } & \multicolumn{2}{|c|}{ No staining } \\
\hline & LV & EV & LV & EV & LV & EV & LV & EV & LV & EV & LV & EV & LV & EV & LV & EV \\
\hline 1 & & & & & * & * & & & & & * & * & & & & \\
\hline 2 & * & * & & * & & & & & * & * & & * & & & & \\
\hline 3 & * & & 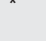 & $x^{*}$ & & & & * & * & & * & * & & & & \\
\hline $\begin{array}{l}4 \\
5\end{array}$ & " & & & & & & * & * & * & & & * & & & & \\
\hline 6 & * & & & * & & & & & & & & * & * & & & \\
\hline 7 & & & & & & & * & * & & & & & & & * & * \\
\hline 8 & * & & & & & * & & & * & & & & & * & & \\
\hline 9 & & & & & * & * & & & & & & & * & * & & \\
\hline 10 & * & & & & 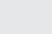 & * & & & & & & & & * & * & \\
\hline 11 & & & & & * & * & & & & & & & * & * & & \\
\hline
\end{tabular}

that reversal of polarity occurs preferentially in the lymphovascular environment. Reversed tumour polarity is of potential importance because it results in exposure of otherwise "hidden" luminal antigens on the external surface of tumour cell groups, allowing interaction between them and the luminal surface of lymphatics and blood vessels. Such interactions could affect the establishment of metastasis and may therefore provide a target for therapeutic intervention. Similar reversal of glandular polarity has recently been identified in the lymphovascular compartment of malignant mesothelioma. ${ }^{4}$

\section{"Basement membrane components are known to direct polarity in normal and neoplastic epithelia, and their apparent absence from the lymphovascular tumour compartment may contribute to the reversed polarity tumour phenotype"}

In a lymphovascular location, release from tumour contact with extravascular connective tissue components may be a factor contributing to the acquisition of reversed glandular polarity. In this context, it is interesting that the basement membrane component laminin was not identified in the lymphovascular tumour compartment, despite its presence in the extravascular compartment of eight of the 11 tumours. Basement membrane components are known to direct polarity in normal and neoplastic epithelia, ${ }^{5-7}$ and their apparent absence from the lymphovascular tumour compartment may contribute to the reversed polarity tumour phenotype.

Although the reversed polarity phenotype was identified in the extravascular tumour compartment in only a single case, it could confer a selective growth advantage to the invading

\section{Take home messages}

- Reversed glandular polarity was seen in invasive ductal breast carcinomas and was significantly more frequent within vessels than outside of them

- Reversal of tumour glandular polarity results in apical domain-type molecules being aberrantly expressed on the external surface of tumour clumps

- This allows direct interaction between these molecules and the lymphovascular endothelium, which may affect the establishment of metastatic disease tumour by the internalisation of basal domain epitopes that would otherwise bind to extracellular matrix and possibly impede tumour invasion. Such reversal of polarity has been described previously in invasive micropapillary breast carcinoma. ${ }^{8-10}$

Given the often focal expression of the polarity markers AUA-1 and HMFG-1 in the breast carcinomas studied, our assessment of the frequency and extent of polarity reversal in these tumours was probably a conservative one. The characterisation and use of other polarity markers with higher tumour expression might reveal greater degrees of tumour polarity reversal. Electron microscopic confirmation of the reversal of tumour polarity within lymphovascular spaces would be useful, but will require a prospective study, which would be hampered by the difficulty associated with the ultrastructural identification of the often tiny lymphovascular space tumour compartments.

Our finding that the endothelium of some vessels containing tumour expressed lower amounts of CD31 suggests that lymphovascular tumour decreases the expression of endothelial CD31. Because CD31 is an interendothelial cell adhesion molecule its downregulation adjacent to lymphovascular tumour, as reported in our present study, may allow easier penetration of the endothelial barrier by tumour cells.

\section{ACKNOWLEDGEMENTS}

We thank Mr RM Watkins (consultant breast surgeon, Derriford Hospital) for his support, Dr S Shaw (Department of Mathematics and Statistics, Plymouth University) for help with the statistical analysis, and Mrs $\mathrm{H}$ Blakely for help in preparation of the manuscript. This work was funded by NHS Regional Grant No RS/ 06/04.99.

\section{Authors' affiliations}

S A Adams, M E F Smith, L A Carr, Department of Histopathology, Derriford Hospital, Derriford Road, Plymouth PL6 8DH, UK G P Cowley, Department of Histopathology, Lincoln County Hospital, Greetwell Road, Lincoln LN2 5QY, UK

Correspondence to: Dr M Smith, Department of Histopathology, Derriford Hospital, Derriford Road, Plymouth PL6 8DH, UK; mark. smith@phnt.swest.nhs.uk

Accepted for publication 12 March 2004

\section{REFERENCES}

1 Cowley GP, Smith MEF. Modulation of E-cadherin expression and morphological phenotype in the intravascular component of adenocarcinomas. Int $J$ Cancer 1995;60:325-9. 
2 Spurr NK, Durbin H, Sheer D, et al. Characterization and chromosomal assignment of a human cell surface antigen defined by the monoclonal antibody AUA-1. Int J Cancer 1986;38:631-6.

3 Heyderman E, Steele K, Omerod MG. A new antigen on the epithelial membrane: its immunoperoxidase localization in normal and neoplastic tissue. J Clin Pathol 1979:32:35-9.

4 Adams SA, Sherwood AJ, Smith MEF. Malignant mesothelioma: PAS/D positivity and inversion of polarity in intravascular tumour. Histopathology 2002;41:1-3.

5 Kirkland S. Polarity and differentiation of human rectal adenocarcinoma cells in suspension and collagen gel cultures. J Cell Sci 1988;91:615-21.

6 Slade MJ, Coope RC, Gomm JJ, et al. The human mammary gland basement membrane is integral to the polarity of human epithelial cells. Exp Cell Res 1999;247:267-78.
7 Pignatelli M, Bodmer WF. Genetics and biochemistry of collagen bindingtriggered glandular differentiation in a human colon carcinoma cell line. Proc Natl Acad Sci U S A 1988;85:5561-5.

$8 \mathrm{Ng}$ WK. Fine-needle aspiration cytology findings of an uncommon micropapillary variant of pure mucinous carcinoma of the breast: review of patients over an 8-year period. Cancer 2002;96:280-8.

9 Luna-More S, Gonzalez B, Acedo C, et al. Invasive micropapillary carcinoma of the breast. A new special type of invasive mammary carcinoma. Pathol Res Pract 1994;66:668-74.

10 Nassar H, Pansare V, Zhang H, et al. Reverse polarization of cells with expression of $\mathrm{MUCl}$ in the stroma-facing surfaces may be a key factor in the pathogenesis and morphogenesis of invasive micropapillary carcinoma. Mod Pathol 2003;16:298A. 